scared to walk on narrow roadsides because of speeding vehicles. They also found crossing the road dangerous because of the lack of designated pedestrian crossings and disregard shown by drivers. Poor visibility caused by random roadside parking and trees also increased the sense of road danger.

Conclusion Children expressed multiple concerns which made the journey difficult and dangerous. Some of these issues could be addressed through improved road infrastructure, whilst others would require a change in driver's behaviour.

Learning Outcomes Children's views are often ignored in issues pertaining to their health and well-being. PEI is an engaging method that encourages children to express concerns about their safety.

\section{A.002 THE EFFECTIVENESS OF BOOSTER SEAT USE IN MOTOR VEHICLE COLLISIONS}

${ }^{1}$ Tona Pitt, ${ }^{2}$ Andrew Howard, ${ }^{1,3}$ Tate HubkaRao, ${ }^{1,3,4,5,6,7}$ Brent Hagel ${ }^{*}$. ${ }^{1}$ Department of Paediatrics, Cumming School of Medicine, University of Calgary, Calgary, Canada; ${ }^{2}$ Departments of Surgery and Health Policy, Management, and Evaluation, Hospital for Sick Children, University of Toronto, Toronto, Canada; ${ }^{3}$ Department of Community Health Sciences, Cumming School of Medicine, University of Calgary, Calgary, Canada; ${ }^{4}$ Faculty of Kinesiology, University of Calgary, Calgary, Canada; ${ }^{5} \mathrm{O}^{\prime}$ Brien Institute of Public Health, University of Calgary, Calgary, Canada; ${ }^{6}$ Sport Injury Prevention Research Centre, University of Calgary, Calgary, Canada; ${ }^{7}$ Alberta Children's Hospital Research Institute, University of Calgary, Calgary, Canada

\subsection{6/injuryprev-2021-safety. 170}

Background Alberta remains the only province in Canada without booster seat legislation. This presents an opportunity to study the effectiveness of booster seats in real-world settings. To date, results of booster seat effectiveness compared with seat-belt-only use has demonstrated mixed findings.

Methods We used Alberta police collision report data from 2010-2016. The study population includes all motor vehicle collisions involving at least one 4-8-year-old. Using a casecontrol study design, children who were reported by police to be injured (cases) were compared with those uninjured (controls) for booster seat use. Logistic regression was used to estimate the association between booster seat use and injury.

Results There were 12,922 children involved in a collision, resulting in 673 child injuries. There were 793 children in the front seat excluded from analysis. Unadjusted analyses indicate that compared with booster seat users, there were greater odds of injury for seat-belt users $(\mathrm{OR}=1.21 ; 95 \%$ CI: $1.02-1.44)$ and those not using any restraints $(\mathrm{OR}=$ 8.16; 95\% CI: 4.66-14.31). When stratifying by impact and collision types, front-end vehicle-on-vehicle collisions demonstrated greater odds of injury for seat-belt wearers relative to those in booster seats $(\mathrm{OR}=1.51$ 95\% CI: 1.10-2.08).

Conclusion Crude analyses indicate a protective effect of booster seats compared with seat-belts, depending on the type of collision and impact location.

Learning Outcomes These regionally-specific injury data may help inform policy on the use of booster seats. Stratification by collision impact location may be necessary to inform analyses on booster seat effectiveness.

\section{B - Injury \& COVID, March 25, 2021}

\section{B.001 TELEHEALTH APPLIED TO DELIVER BEHAVIORAL SKILLS TRAINING TO REDUCE CAR SEAT MISUSE}

'James M James Decarli*. 'Pro Consumer Safety/public Health Behavior Solutions, Los Angeles, USA; ${ }^{2}$ Occupant Protection, California Strategic Highway Safety Plan, Sacramento, USA

10.1136/injuryprev-2021-safety. 171

Telehealth is a common approach to deliver health education before and during the COVID-19 pandemic. However, its ability to apply behavioral skills training (BST) to teach parents how to install and use their child restraint system (CRS) has been undocumented. This session will review how telehealth was used to deliver behavioral skills training (BST) to teach 171 expectant parents, in a multiple-baseline-acrosssubject design, how to install and use their CRS in order to reduce misuse and improve retention. While baseline results identified significant misuse across all participants. Once BST was delivered by the use of telehealth, misuse improved by $97 \%$ for 37 task objectives. During the 2-week follow-up evaluation concluded that $100 \%$ of participants retained the skills they mastered during BST. This session will describe the validation of telehealth as an effective method of delivering BST to car seat education to expectant parents during COVID-19 and illustrate how telehealth has broader CRS program and train-the-trainer implications beyond the COVID-19 pandemic.

\section{B.002 TRANSPORT AND HEALTH DURING AND AFTER THE COVID-19 PANDEMIC}

1,2,3 Olive Kobusingye* ${ }^{*}, 3$ Margie Peden. ${ }^{1}$ Road Traffic Injury Research Network, Kampala, Uganda; ${ }^{2}$ Makerere University School of Public Health, Kampala, UGANDA; ${ }^{3}$ The George Institute for Global Health, Sydney, Australia

10.1136/injuryprev-2021-safety. 172

In May 2020, the High Volume Transport (HVT) Applied Research Programme which is funded by UKAid through the Foreign, Commonwealth \& Development Office (FCDO), published an insight paper by the Road Traffic Injury Research Network's RTIRN Chair and Deputy Chair (Drs Kobusingye and Peden) on the nexus between transport and health during the COVID-19 pandemic.

The report looks at the five interacting dimensions between transport and public health - safety, active transport, clean air, connectivity/access, and equity - during COVID-19. It highlights the positive unintended effects of the pandemic - reductions in road crashes and improvements in air quality - and questions what can be done to maintain some of these gains.

The report emphasises the gaps in current knowledge, particularly in low- and middle-income countries, such as the effectiveness of masks in crowded public transport, and the impact of severe transport restrictions on vulnerable populations, especially older people, adolescents, pregnant women, and persons with disabilities. It argues that COVID-19 offers an opportunity for an inter-sectoral response from the health and transport sectors to build safer, more equitable, and resilient transport systems.

Transport systems were responsible for converting a local epidemic into a pandemic, and they have been central to the 